\title{
Risk assessment of the subclinical hypothyroidism in non- alcoholic fatty liver disease patients with different levels of cardiovascular risk
}

\author{
Olena Kolesnikova ${ }^{1}$, Valeriya Nemtsova ${ }^{2}$ and Anna Isayeva ${ }^{1}$ \\ ${ }^{1}$ Government Institution «L.T.Malaya Therapy National Institute of the National Academy of Medical Sciences of Ukraine, Kharkiv, Ukraine \\ ${ }^{2}$ Kharkiv National Medical University, Kharkiv, Ukraine
}

\begin{abstract}
Objective:_To assess the risk of developing subclinical hypothyroidism (SH) in patients with non-alcoholic fatty liver disease (NAFLD) with a different risk of cardiovascular complications, and to identify the link between high risk of developing SH and major cardiovascular factors ( $\mathrm{CV}$ ). Material and methods: A one-stage clinical trial included 298 people with NAFLD with the presence of one or more CV risk factors (arterial hypertension, smoking, hypercholesterolemia) and various levels of CV risk factors on the SCORE scale. All patients were questioned on a standard questionnaire and HRQ, a thyroid status study was conducted.

Result: Patients were divided into three groups according to the level of total CV risk for SCORE: $33.9 \%$ had low / moderate CV risk, $41.9 \%$ - high and $24.2 \%$ - very high CV risk. The majority of the subjects were in the age groups 50-59 and 60-69 years. In the age group 40-49 years, $22.2 \%$ of patients had high CV risk, $28 \%$ of men aged 50-59 years had moderate CV risk, while $49.7 \%$ of older patients showed very high CV risk. According to the results of a survey on the HRQ questionnaire, a low risk of developing hypertension was found in 34.9\%, a moderate risk in $48.9 \%$, and a high risk was found in $16.1 \%$ of patients. Persons with a very high $\mathrm{CV}$ risk on the SCORE scale also have a high risk of developing the SH according to the HRQ questionnaire. Among patients with the presence of one or more of the above $\mathrm{CV}$ risk factors, early markers of thyroid status disorders should be identified and the risk of developing hypertension should be assessed. Such a tactic contributes to an adequate assessment of the risk of SH in patients with NAFLD and the development of methods for primary prevention.
\end{abstract}

\section{Introduction}

Increased interest in subclinical hypothyroidism $(\mathrm{SH})$ is due to the fact that the prevalence of this disease is much higher than the prevalence of manifested hypothyroidism [1]. There are few publications that the presence of $\mathrm{SH}$ in the patients with nonalcoholic fatty liver disease (NAFLD), on the one hand, may increase its course, and, on the other hand, NAFLD promotes the hypofunction of the thyroid gland, thereby participating in the formation of cardiovascular risk (CVR) in this category of patients.

Opinions about the active SH screening of experts from different medical communities are controversial. Some tend to behave aggressive tactics in conducting screening from 35 years of age, every 5 years among high-risk groups, others such as the Royal College of Physicians and U.S. Preventive Services Task Force are limited by the fact that there is insufficient data for or against screening, therefore the active screening is not recommended [2].

Due to the lack of large randomized controlled trials (RCTs) for assessing the clinical consequences of $\mathrm{SH}$, the available screening and treatment recommendations are based on observational and small clinical trials and also expert opinions. Prospective studies have shown an increase in the risk of cardiovascular coronary events, heart failure and cardiovascular mortality among patients with $\mathrm{SH}$, which determines the relevance of the studying this problem.

Traditionally, for the detection of early thyroid function disorders, a study of thyroid hormones is performed. However, in recent years, the use of questionnaires to detect (screening) early markers of thyroid gland dysfunction is suggested. Thereby, the purpose of this study was to assess the risk of SH development in NAFLD patients with varying risk of cardiovascular complications, as well as the relationship between high risk of SH development and the main factors of CVR.

\section{Materials and methods}

The study was based on the results of a questionnaire and clinical examination of 298 subjects aged 40-69 who applied to outpatient department of the GI «L.T. Malaya Therapy National Institute of the National Academy of Medical Sciences of Ukraine», Kharkiv, Ukraine for any reason with an obligatory indication in medical history the presence of NAFLD. The study consisted of several stages. At the first stage, medical documents were analyzed in order to assess the availability of risk factors and the calculation of the total CVR on the SCORE scale. In the second stage, 298 respondents were divided into CVR groups: low and moderate ( $0-4 \%$ on the SCORE scale), high (5-

Correspondence to: Olena Kolesnikova, L.T. Malaya Therapy National Institute of the National Academy of Medical Sciences of Ukraine, Kharkiv, Ukraine; E-mail: kolesnikova1973@gmail.com

Key words: subclinical hypothyroidism; non-alcoholic fatty liver disease; early thyroid status disorders; cardiovascular risk

Received: September 28, 2017; Accepted: October 19, 2017; Published: October 23,2017 
$9 \%$ on the SCORE scale) and very high (> $10 \%$ on the SCORE scale) without clinical manifestations of CVD.

Inclusion criteria for the study were the presence of one or more risk factors from the following: arterial hypertension $(\mathrm{AH}) 1-3$ degrees, smoking, hypercholesterolemia [total cholesterol (TC) $>5 \mathrm{mmol} / \mathrm{l}$ ], excess body weight or obesity.

Exclusion criteria: manifested hypothyroidism; symptomatic $\mathrm{AH}$; diabetes; presence of cerebrovascular diseases; heart disease (clinically expressed IHD, history of myocardial infarction, coronary revascularization, chronic heart failure III- IV stage by NYHA); renal and hepatic insufficiency; oncology; rheumatic diseases; allergic and autoimmune diseases; pregnancy; unwillingness of the patient to participate in the study.

At the third stage, to determine the risk of $\mathrm{SH}$ developing all participants were interviewed using the Hypothyroid Risk Questionnaire (HRQ) questionnaire [3, 11].

At present, in Ukraine this available method for predicting the risk of SH development is not applicable. Obviously, this is due to the lack of clinical data on the feasibility of using the questionnaire in practical medicine, in particular, among those with CVD and SH risk factors. To increase the size of the groups and to analyze the total score obtained on the SCORE scale and the HRQ questionnaire, the patients were divided into groups according to the HRQ questionnaire: low risk of $\mathrm{SH}$ development (sum of points on the scale $<10$ ), moderate risk (sum of points 10-20), high/very high risk (sum of points $>20$ ); on the SCORE scale: low/moderate risk (0-4\%), high (5-9\%), very high (>10\%).

Anthropometric parameters with an estimation of growth with an accuracy of $0.5 \mathrm{~cm}$; body weight with an accuracy of $0.1 \mathrm{~kg}$; Kettle index (as the ratio of body weight in kilograms to square of height in meters); waist circumference accurate to $0.5 \mathrm{~cm}$ were studied in all patients. Blood pressure was measured by a mechanical tonometer on the right arm with an accuracy of $2 \mathrm{~mm} \mathrm{Hg}$. twice with a 5-minute interval in a sitting position at rest. For analysis the mean value of two measurements was used. Biochemical tests were conducted in the morning on an empty stomach after a 12-hour fasting period from blood taken from the elbow vein. The level of total cholesterol, triglycerides (TG), cholesterol of high lipoprotein (HDL cholesterol) and low (LDL cholesterol) density, a fasting glucose level in plasma of venous blood were determined. The concentration of thyroid hormone (THS), free thyroxine (T4 free) and thyroid peroxidase antibodies (TPO-AB) in serum was investigated by immunoassay analysis (kits of SPL “Granum", Ukraine).

The statistical analysis was performed using the statistical package SPSS 17.0. In this study $\chi 2$, the Student t-test and Fischer's criterion (F-test) were used. When constructing logistic regression, nonmodifying risk factors of the SCORE scale and the HRQ questionnaire were taken into account.

\section{Result}

According to the protocol all patients were divided into three groups in accordance with the level of total cardiovascular risk by SCORE scale: $33.9 \%$ (101 patients) had low/moderate risk, 41.9\% (125 patients) had high and $24.2 \%$ (72 patients) very high risk. In addition, patients were divided into three age groups: the first group was 40-49 years, the second group was 50-59 years, the third group 60-69 years. Among the subjects with high CVR were mostly individuals aged 60-69 years $(34.6 \%)$ and patients aged $50-59(30.2 \%)$, persons younger than
50 years old accounted for $9.4 \%$. In the group of very high CVR in approximately equal proportions were patients aged 50-59 (19.8\%) and 60-69 (23.5\%) years.

The probability of SH development in different age groups among the patients with different CVR risk was analyzed. In general, positive associations of the risk of $\mathrm{SH}$ development with age were revealed $(\mathrm{r}=$ $0.42 ; \mathrm{p}=0.001)$. Thus, in the age group of $40-49$ years a very high risk of SH was observed in $22.2 \%$ of patients, at the age of $50-59$ - in $28 \%$, while in the 60-69 age group a very high risk of SH was detected in $49,7 \%$ of patients. In the older age group, the number of patients with low and moderately increased risk of SH development is statistically significantly lower compared with younger patients $(p=0.001)$.

The analysis of the results shows that among the patients with different levels of cardiovascular risk by the SCORE scale, in general, $29,07 \%$ of patients showed a high risk of SH development according to the HRQ questionnaire (Table 1). The highest number of patients with low SH risk were observed in patients with low/moderate cardiovascular risk (59.8\%), while in groups with high and very high cardiovascular risk, the probability of $\mathrm{SH}$ development was $32 \%$ and $14 \%$, respectively.

$37.9 \%$ of the patients with low/moderate CVR, 51.2\% with high CVR and 56.98\% with very high CVR a moderate risk of SH development was demonstrated. Among the patients with high risk of SH development the majority were those with very high CVR (29.07\%), which significantly exceeds the comparison of patients with high and low / moderate CVR: $16.8 \%$ and $2.3 \%$, respectively.

In addition, a number of features in the definition of the relationship between separate $\mathrm{CV}$ risks with high and very high risk of SH development were revealed (Table 2). A significant part of smokers was in the group of very high CVR with high risk of SH development ( $18.6 \%$ vs. $1.2 \%$ and $4.2 \%$ in the first and second groups, respectively). Hypertension was associated with a high incidence in groups of high and very high CVR in SH high-risk subjects: $78 \%$ and $82.4 \%$, respectively, against the group with a low CVR - 24.6\%. The proatherogenic lipid profile (increase of total cholesterol, LDL cholesterol and decrease of HDL cholesterol) was prevalent in SH high-risk groups in patients with very high CVR.

Fasting hyperglycemia (FH) was dominated in a group of patients with a very high risk of cardiovascular complications. The sedentary lifestyle was noted in the groups of high and very high CVR $(26.0 \%$ and $32.2 \%$, respectively). Similar tendencies, but more pronounced, with the incidence of overweight and obesity (abdominal) were observed: in the high-CVR group BMI $>25 \mathrm{~kg} / \mathrm{m} 2$ was observed in $22 \%$ of patients, in a very high - CVR group- $37.2 \%$. At the same time, waist $>94 \mathrm{~cm}$ was registered in almost half of patients with very high CVR.

One of the tasks of the study was to detect early violations of thyroid status among the people with different levels of CVR by the SCORE scale. The presence of hypothyroidism in the immediate relatives was observed in $7.6 \%$ of patients with low/moderate CVR, $18.5 \%$ and $24.2 \%$ in the groups of high and very high CVR, respectively.

An early violation of the thyroid status was detected among people with different CVRs, (Table 3). A detailed analysis showed that in the group of low/moderate cardiovascular risk in $20,9 \%$ of patients an increased level of TSH in the absence of reduction of T4 were detected. In the group with high CVR in 36\% an increased TSH levels were found and $1,2 \%$ of patients showed a decrease in T4 levels. 
Table 1. Detection of different degrees of risk of SH development by the HRQ questionnaire among patients with low, moderate and high risk by the SCORE scale

\begin{tabular}{|c|c|c|c|}
\hline \multirow{2}{*}{ CVR by SCORE scale } & \multicolumn{3}{|c|}{ Risk of SH development } \\
\cline { 2 - 4 } & Low & Moderate & High \\
\hline Low/Moderate & $52(59,8 \%)$ & $33(37,9 \%)$ & $2(2,3 \%)$ \\
\hline High & $40(32 \%)$ & $64(51,2 \%)$ & $21(16,8 \%)$ \\
\hline Very high & $12(14 \%)$ & $49(56,98 \%)$ & $25(29,07 \%)$ \\
\hline Total & $104(34,89 \%)$ & $146(48,99 \%)$ & $46(16 \%)$ \\
\hline
\end{tabular}

Table 2. High risk of SH development among the patients with risk factors in three groups by the SCORE scale

\begin{tabular}{|c|c|c|c|c|c|c|}
\hline \multirow[t]{2}{*}{ Risk factors } & \multicolumn{2}{|c|}{ Low/moderate CVR/ High-HRQ risk } & \multicolumn{2}{|c|}{$\begin{array}{c}\text { High CVR/ } \\
\text { High-HRQ risk }\end{array}$} & \multicolumn{2}{|c|}{ Very high CVR/ High-HRQ risk } \\
\hline & SCORE & HRQ & SCORE & HRQ & SCORE & HRQ \\
\hline Smoking, \% & 3,6 & 1,2 & 10,4 & 4,2 & 22,5 & 18,6 \\
\hline $\mathrm{BP}, \mathrm{mm} . \mathrm{Hg}$. & 12,8 & 24,6 & 56,3 & 78,0 & 68,2 & 82,4 \\
\hline $\mathrm{BMI}>25 \mathrm{~kg} / \mathrm{m}^{2}$ & 3,6 & 10,2 & 11,8 & 22,0 & 24,6 & 37,2 \\
\hline Waist $>94 \mathrm{sm}$ & 4,2 & 11,8 & 11,8 & 24,2 & 22,9 & 48,4 \\
\hline The sedentary lifestyle, $\%$ & 4,2 & 1,2 & 11,8 & 26,0 & 24,6 & 32,2 \\
\hline Heredity for hypothyroidism, $\%$ & 1,2 & 7,6 & 7,6 & 18,6 & 3,6 & 24,2 \\
\hline $\mathrm{TC}>5 \mathrm{mmol} / 1$ & 3,6 & 4,2 & 12,8 & 10,2 & 32,2 & 24,6 \\
\hline LDL cholesterol $>3 \mathrm{mmol} / 1$ & 3,6 & 7,6 & 12,8 & 10,4 & 24,6 & 32,2 \\
\hline HDL cholesterol $<1,0 \mathrm{mmol} / 1$ & 3,6 & 1,2 & 10,4 & 11,8 & 22,9 & 37,2 \\
\hline $\mathrm{FG}>5,6 \mathrm{mmol} / 1$ & 4,2 & 11,8 & 10,4 & 12,8 & 18,6 & 22,9 \\
\hline
\end{tabular}

Table 3. Violation of thyroid status among people with different levels of CVR by the SCORE scale

\begin{tabular}{|c|c|c|}
\hline CVR by the SCORE scale & Increase of TSH & Decrease T4 \\
\hline Low/moderate, $\mathrm{n}(\%)$ & $18(20,9 \%)$ & $0(0)$ \\
\hline High, $\mathrm{n}(\%)$ & $31(36 \%)$ & $1(1,2 \%)$ \\
\hline Very high, $\mathrm{n}(\%)$ & $37(43 \%)$ & $2(2,3 \%)$ \\
\hline
\end{tabular}

In patients with very high CVR an increase number of patients with increased basal TSH were observed. The presence of the T4 reduction in $2.3 \%$ of patients, as in the high CVR group, is probably due to the fact that hypothyroidism is already manifest at the time of the study.

\section{Discussion}

The interest in early detection of high-risk groups of $\mathrm{SH}$ development in patients with NAFLD with different levels of CVR is due to a number of causes.

On the one hand, it was shown that $\mathrm{SH}$ is associated with an increased risk of fatal and nonfatal coronary heart disease (CHD) [4]. Data of the analysis of 55,000 CHD patients had shown a confidence interval of 1.89 (95\% CI 1.28-2.80) for coronary heart disease events in TSH levels above $10 \mathrm{mIU} / 1$ [5] compared with euthyroidism and 1, 58 (95\% CI 1.10-2.27) for fatal CHD. These risks did not increase in subjects with TSH lower than $7 \mathrm{mIU} / \mathrm{L}$, the mortality from all causes not increased with an increase in TSH. An increase of CVR associated with an increase in TSH levels above $10 \mathrm{mIU} / \mathrm{L}$ can be explained by several reasons. At $\mathrm{SH}$ levels of cholesterol increases and dyslipidemia develops [6]. A systematic review of 13 heterogeneous studies has demonstrated the effect of substitution therapy with Levothyroxine at $\mathrm{SH}$ on reducing the concentrations of LDL cholesterol and total cholesterol [7]. Similar results have been demonstrated in other RCTs [8].

In addition, deserves attention data from an interventional study of 56 women with $\mathrm{SH}$, in which an increase in systolic and diastolic pressure, serum concentrations of TC and homocysteine in patients compared with the healthy control group were found with followed by normalization of these parameters after 18 months of substitution therapy with Levothyroxine $[4,7,9]$. The obtained data confirm the presence of a connection between $\mathrm{SH}$ and CVR.
On the other hand, high prevalence of a metabolic syndrome (MS) components, including such a pathological state as NAFLD, independently determines CVR in the presence of the latter. Given the fact that according to prospective cohort studies an increase in the prevalence of MS components among adults with $\mathrm{SH}$ is observed [9], there is reason to assume potentiating influence on the formation of CVR both NAFLD and SH. The increasing of arteries intima-media thickness [6], predisposition to hypercoagulation, insulin resistance, oxidative stress [9] and endothelial dysfunction [5] also contributes to increase a CVR in SH. On the background of substitution therapy with Levothyroxine in patients with $\mathrm{SH}$ a decrease of carotid arteries intima-media thickness [6], an improvement of endothelial function [5], improvement of cardiac function [4] were observed. Such therapy may reduce the risk of coronary heart disease in relatively young patients with $\mathrm{SH}$, but not in people aged over 70 years [8]. Adequate RCTs devoted to the study of cardiovascular events in the renew of euthyroidism is still few.

The confirmation of the contribution of both SH and NAFLD to the formation of CVR are the results of the potential association between SH and NAFLD that were obtained by Chung et al., who revealed a dose-dependent relationship between TSH levels and severity of NAFLD in patients with SH [10].

Hence, the detection of high SH risk among NAFLD patients with varying degrees of CVR is of the great importance in the primary prevention of CVD. For this purpose, we used the HRQ questionnaire. It is known that this method belongs to the category of routine screening method for assessing the risk of SH. Of course, it has an applied value, first of all in general therapeutic practice, since the patients with early markers of thyroid status disorders are found predominantly among patients with risk factors for cardiovascular complications. This group, in the first place, includes persons with hypertension, overweight and obesity, dyslipidemia, MS and NAFLD.

Thus, we have studied the possibility of using the HRQ questionnaire in the general therapeutic practice. On the other hand, we conducted an analysis of the combination of CVR with the risk of $\mathrm{SH}$ development in NAFLD patients. The detection of regularities 
allows to determine the tactics of primary prophylaxis for $\mathrm{SH}$ and CVD at the same time in patients with NAFLD. In this study among patients with high risk of $\mathrm{SH}$ development and different cardiovascular risk levels in $17 \%$ a very high CVR and in $29 \%$ high CVR were occurred. It is obvious that it is necessary to realize a primary prophylaxis of $\mathrm{SH}$ in this category of patients, since the conversion of early violations of the thyroid status into manifested hypothyroidism increases the CVR with a fatal outcome in 2-3 times. Among the persons with moderate risk of SH development the high frequency of high CVR - 51\% and very high CVR - 57\% were occurred.

In accordance with the obtained data it is also necessary to conduct a primary prevention of cardiovascular events in persons with moderate risk of $\mathrm{SH}$.

The study of the relationship between moderate and high risk of SH developing by the HRQ questionnaire and individual risk factors showed that smoking, sedentary lifestyle, hypercholesterolemia and low HDL levels are associated with a high risk of SH developing, whereas $\mathrm{AH}$ is associated with both moderate and high risk of $\mathrm{SH}$.

It should be emphasized that according to the results of the study the patients with elevated TSH had high (36\%) and very high (43\%) CVR. The obtained data are consistent with the results presented in the meta-analysis of N. Rodondi, which combined the data of more than 55 thousand patients and demonstrated that the risk of CVD and mortality correlated with elevated levels of TSH [4].

\section{Conclusion}

The assessment of the risk of SH development in NAFLD patients with separation of high-risk group is an important stage in primary prevention. According to this study, people with a very high risk by the SCORE scale also have a moderate and high risk of SH developing according to the HRQ questionnaire. Risk factors such as smoking, hypertension, dyslipidemia, sedentary lifestyle and obesity have a close association with the high risk of SH in NAFLD patients.
Thus, in patients with the presence of one or more of the above listed risk factors, early markers of thyroid status disorders and an evaluation of the risk of SH development should be identified. This tactic will help to adequately assess the risk of SH in NAFLD patients and develop prevention methods.

\section{References}

1. Dreval AV (2016) Endocrinology: a guide for doctors. Moscow: GEOTAR-Media. 148-157.

2. Chung GE, Kim D, Kim W, Yim JY, Park MJ, et al. (2012) Non-alcoholic fatty liver disease across the spectrum of hypothyroidism. J Hepatol 57: 150-156. [Crossref]

3. Liu D, Jiang F, Shan Z, Wang B, Wang J, et al. (2010) A cross-sectional survey of relationship between serum TSH level and blood pressure. J Hum Hypertens 24: 134138. [Crossref]

4. Walsh JP, Bremner AP, Bulsara MK, O’Leary P, Leed-man PJ, et al. (2006) Subclinica thyroid dysfunction and blood pressure: a community-based study. Clin Endocrinol 65 486-491. [Crossref]

5. Razvi S, Weaver JU, Butler TJ, Pearce SH (2012) Levothyroxine treatment of subclinical hypothyroidism, fatal and nonfatal cardiovascular events, and mortality. Arch Intern Med 172: 811-817. [Crossref]

6. Müller B "Subklinische" Hypo- und Hyperthyreose. Schweizerische Gesellschaft für Endokrinologie und Diabetologie.

7. Monzani F, Caraccio N, Kozakowa M, Dardano A, Vittone F, et al. (2004) Effect of levothyroxine replacement on lipid profile and intima-media thickness in subclinical hypothyroidism: a double-blind, placebo-controlled study. J Clin Endocrinol Metab 89: 2099-2106. [Crossref]

8. Rodondi N, den Elzen WP, Bauer DC, Cappola AR, Razvi S, et al. (2010) Subclinica hypothyroidism and the risk of coronary heart disease and mortality. JAMA 304: 13651374. [Crossref]

9. Razvi S, Ingoe L, Keeka G, Oates C, McMillan C, et al. (2007) The beneficial effect of L-thyroxine on cardiovascular risk factors, endothelial function, and quality of life in subclinical hypothyroidism: randomized, crossover trial. J Clin Endocrinol Metab 92: 1715-1723. [Crossref]

10. Hollowell JG, Staehling NW, Flanders WD, Hannon WH, Gunter EW, et al. (2002) Serum TSH, N4, and thyroid antibodies in the United State population (1988 to 1994): National Health and Nutrition Examination Survey (NHANES III). J Ckin Endocrinol Metab 12: 839-847. [Crossref]

11. Hypothyroid Risk Questionnaire ${ }^{\mathrm{TM}}$ by Joseph J. Collins, RN, ND. Available from: https://www.yourhormones.com/

Copyright: (C2017 Kolesnikova O. This is an open-access article distributed under the terms of the Creative Commons Attribution License, which permits unrestricted use, distribution, and reproduction in any medium, provided the original author and source are credited. 